\title{
The efficiency of tactical and technical actions of the national teams of Japan and Russia at the World Championships in Judo (2013, 2014 and 2015)
}

\author{
Marek Adam ${ }^{1}$, Katarzyna Sterkowicz-Przybycieñ ${ }^{2}$ \\ ${ }^{1}$ Department of Combat Sports, University of Physical Education and Sport, Gdańsk, Poland; ${ }^{2}$ Department of Gymnastics \\ and Dance, Institute of Sport Science, University of Physical Education, Kraków, Poland
}

\section{Summary}

Study aim: The aim of the study was to determine the value of indices characterizing the efficiency in attack and in defence in Judo influencing the course and the outcome of Japanese and Russian representatives' fights.

Material and methods: Research material comprised the results of observations and an analysis of bouts of Japanese ( $\mathrm{n}=15$, 124 bouts) and Russian ( $\mathrm{n}=16,103$ bouts) athletes during the World Championships in 2013, 2014 and 2015. The observed athletes' bouts were with different opponents. The collected material allowed determination of the value of the efficiency in attack indices (Sa) of groups of judo techniques and the indices in defence (So) characterising Japanese and Russian representatives' opponents. The techniques dominant among the observed athletes were identified.

Results: The Japanese were characterised by significantly higher values of the efficiency in attack indices resulting from throws, in particular, foot throws. The efficiency in defence in both teams was significantly lower when applying holds rather than throws. Representatives of Russia also had high efficiency in applying joint locks and low efficiency in defensive actions.

Conclusion: Sports results obtained by representatives of both teams were connected not only with the efficiency of their defensive actions, but also with the type and efficiency of the techniques dominant in attack.

Key words: Analysis of fight - Technical-tactical preparation - Indices - Sports competition - Leading teams

\section{Introduction}

The factor of judo athletes' technical-tactical preparation has the largest contribution in generating victory [12, 20, 21, 22, 28]. Hence, more and more often researchers are observing, documenting, analysing and assessing the course of bouts during top-level tournaments. Effects of such research are important for both the theory and the practice of judo, as the statistical findings should result in practical applications $[19,25]$. The obtained information allows monitoring of the training programmes and further individuation of the tactical and technical preparation of athletes of this discipline, which can raise the efficiency of preparation for subsequent stages of the sports competition. Our previous studies also allowed certain distinctive features of Japanese and Russian competitors' training to be distinguished on the basis of efficient attacks performed during judo competitions [2, 3, 4].

After the Olympic Games in London (2012), which turned out to be a great success for Russian judo competitors, preparations for the Olympic Games in Rio de Janeiro (2016) began. The next three World Championships were the main qualification criterion for the Olympics (2016): 26-31 Aug. 2013 in Rio de Janeiro (BRA), 25-30 Aug. 2014 in Chelyabinsk (RUS) and 24-29 Aug. 2015 in Astana (KAZ). The World Championships results show that the representatives of Japan regained the dominant position in the world in men's judo (winning eight gold, three silver and four bronze medals), while Russian representatives failed to win a gold medal (they won four silver and seven bronze medals) [33]. 
It has been assumed that the most interesting indices of technical-tactical preparation that affect the result and the showmanship of bouts will be those that regard attack and defence [3]. Therefore, the aim of the study was: 1) to quantify the efficiency in attack and defence in the Japanese and Russian teams, 2) to compare the level of the efficiency in attack and defence between Japanese and Russian athletes, 3) to identify the dominant techniques in both teams.

\section{Material and methods}

We calculated the sample size (N1 and N2) necessary to demonstrate significant inter-group differences. Expecting large inter-group differences, the parameters adopted in our calculations were as follows: tails two, estimated large effect size, alpha $<0.05$, power $=0.80$, critical $\mathrm{t}=2009$, required sample size group $1: \mathrm{N}=26$, required sample size group $2: \mathrm{N}=26$. In practice, 124 duels of Japanese athletes (JPN, $\mathrm{n}=15$ ) and 103 duels of Russian athletes (RUS, $\mathrm{n}=16$ ) with different opponents at the World Championships in 2013, 2014 and 2015 were registered and analysed. Japanese judo competitors efficiently performed 127 attacks, while their opponents performed 22 efficient attacks. Russian athletes efficiently executed 78 attacks, while their opponents executed 35 effective attacks. The breakdown of techniques was based on the traditional classification of Kodokan Judo [13, 17]. Naming and spelling was adopted according to the dictionary of Kodokan judo [18]. Registration of fights was done by audio-video means (Sony HDR-CX450B), by PZJudo (Polish Judo Association) coaches and then, during playback of the recording from a digital camera, efficient technical-tactical elements were recorded graphically [2]. The collected material allowed for an analysis of fights and calculating selected indices: the efficiency in attack $(\mathrm{Sa})$ and the efficiency in defence So $[1,3]$. The observations were made in accordance with a reliable methodology of registering and analysing judo fights during sports competitions [9, 10, 14, 32].

Based on the collected material, the value of the efficiency in attack index ( $\mathrm{Sa}$ ) was calculated for different groups of judo techniques, while another index (K) allowed us to define a hierarchy of attack techniques which were recorded during the analysed championships.

The efficiency in attack index $(\mathrm{Sa})$ was defined on the basis of the formula:

$$
\mathrm{Sa}=(\mathrm{M} \times 10+\mathrm{M} \times 7+\mathrm{M} \times 5) / \mathrm{n}
$$

where: $\mathrm{Sa}=$ the efficiency in attack index; $\mathrm{M}=$ the number of attacks: assessed with the ippon score (10 points), assessed with the waza ari score (7 points), assessed with the yuko score ( 5 points); $\mathrm{n}=$ the number of analysed bouts.
Similarly to Sa, the efficiency in defence index (So) was defined, taking into consideration opponents' attacks and points obtained by them.

The order of dominant judo techniques was based on three criteria, as defined by the formula:

$$
\mathrm{K}=\mathrm{K} 1+\mathrm{K} 2+\mathrm{K} 3
$$

where: $\mathrm{K}=$ classification of dominant judo techniques during competitions; $\mathrm{K} 1$ = classification of techniques based on the number of ippon scores; K2 = classification of techniques based on the number of efficient attacks (given points by judges); K3 = classification of techniques based on auxiliary points for efficiently performed techniques (ippon -10 points, waza ari -7 points, yuko -5 points).

\section{Statistics}

On the basis of individual Sa and So indices, the group characteristics were calculated (arithmetic mean, standard deviation (SD), median, min-max) for Japanese (JPN, $\mathrm{n}=15$ ) and Russian (RUS, $\mathrm{n}=16$ ) teams. Standardized values of kurtosis and skewness which fall in the range from -2 to 2 were adopted as the criterion of normal distribution for each variable. In inter-group comparisons, the parametric test (ANOVA) or the non-parametric KruskalWallis test was chosen, and the eta-squared effect size value was calculated: 0.01 - small, 0.06 - medium, 0.14 - large. In the case of paired comparisons, we applied respectively the t-test or non-parametric Mann-Whitney test and r effect size: 0.10 - small, 0.24 - medium, 0.37 - large, which is subject to conversion and is equivalent to $\mathrm{d}$ effect size [11]. Calculations were made in STATGRAPHICS Centurion v. 17.

\section{Results}

\section{Comparison of the quality of techniques in attack}

JPN and RUS representatives significantly differed in terms of the quality of performing attacks with use of throws and holds $(\mathrm{F}=17.47, \mathrm{p}<0.001$, eta-squared $=0.48$ [large effect]).

Table 1 shows numeric data whose comparison showed a significant advantage, as measured by the value of $\mathrm{Sa}$, of JPN athletes over the RUS ones in the quality of throws $(\mathrm{t}=2.269, \mathrm{p}=0.031, \mathrm{r}=0.38$ [large effect]). However, no significant intergroup difference was found in the Sa value for katame waza $(\mathrm{t}=0.182, \mathrm{p}=0.856, \mathrm{r}=0.04)$.

Within the 5 subgroups of nage waza there were important differences in $\mathrm{Sa}(\mathrm{H}=55.0, \mathrm{p}<0.001$, etasquared $=0.15$ [large effect]). JPN athletes were characterised by a marked advantage over RUS ones in ashi waza $(\mathrm{W}=66.5, \mathrm{p}=0.035, \mathrm{r}=0.40$ [large effect]) and in te waza $(\mathrm{W}=94.0, \mathrm{p}=0.284, \mathrm{r}=0.27$ [medium effect]). A reverse direction of the difference, however, indicated a trend to 
Table 1. Scoring for nage waza and katame waza (Sa)

\begin{tabular}{lccc}
\hline Techniques & Mean & Median & SD \\
\hline Nage waza & & & \\
\hline JPN & $\mathbf{5 . 5 9}$ & $\mathbf{5 . 4 0}$ & $\mathbf{2 . 3 0}$ \\
RUS & $\mathbf{3 . 5 5}$ & $\mathbf{4 . 4 2}$ & $\mathbf{2 . 6 8}$ \\
\hline Katame waza & & & \\
\hline JPN & 1.26 & 1.43 & 1.14 \\
RUS & 1.18 & 1.26 & 1.29 \\
\hline
\end{tabular}

The bold type shows significant intergroup in nage waza.

a higher quality of yoko sutemi waza throws made by RUS rather than JPN athletes $(\mathrm{W}=155.0, \mathrm{p}=0.063, \mathrm{r}=-0.24$ [medium effect]). A similar phenomenon, but to a lesser extent, occurred in the quality of koshi waza $(\mathrm{W}=143.0$, $\mathrm{p}=0.267, \mathrm{r}=-0.23$ [small effect]), but not in the case of ma sutemi waza, where an advantage was found in JPN judo competitors $(\mathrm{W}=97.5, \mathrm{p}=0.174, \mathrm{r}=0.10$ [small effect]) (Table 2).

Significant differences in the efficiency of three groups of katame waza were observed between JPN and RUS $(\mathrm{H}=18.6, \mathrm{p}=0.002$, eta-squared $=0.13)($ Table 3$) . \mathrm{JPN}$ athletes showed a greater advantage over the RUS ones in the efficient execution of osaekomi waza $(\mathrm{t}=1.61$, $\mathrm{p}=0.117, \mathrm{r}=0.28$ [medium effect]), but not in kansetsu waza $(\mathrm{W}=153.0, \mathrm{p}=0.122, \mathrm{r}=-0.35$ [medium effect] $)$ or in shime waza $(\mathrm{W}=120.0, \mathrm{p}=0.962, \mathrm{r}=-0.03)$.

\section{Comparison of the quality of techniques executed in defence}

Comparisons of the quality of techniques (using the So index) executed in defence against nage waza and katame waza attacks are presented in Table $4(\mathrm{H}=32.149$, $\mathrm{p}<0.001$, eta-squared $=0.47$ [large effect]). As in the case of $\mathrm{Sa}$, in both national teams the efficiency in defence (So) was significantly higher for nage waza than for katame waza. No significant differences were noted, however, in nage waza or katame waza between JPN and RUS $(\mathrm{t}=-1.204, \mathrm{p}=0.238, \mathrm{r}=-0.22)$.

Table 4 shows the quality of defence of JPN and RUS teams measured with the So index during the opponents' attack using nage waza techniques ( $\mathrm{W}=143.0, \mathrm{p}=0.372$, $\mathrm{r}=-0.21$ [small effect]) and katame waza techniques ( $\mathrm{W}=128.0, \mathrm{p}=0.563, \mathrm{r}=-0.14$ [small effect]).

A post-hoc test using the Mann-Whitney test showed significant differences between nage waza JPN and katame waza JPN (W=26.0, $\mathrm{p}<0.001, \mathrm{r}=0.84$ [large effect]) and between nage waza RUS and katame waza RUS ( $\mathrm{W}=30.5, \mathrm{p}<0.001, \mathrm{r}=0.77$ [large effect]). The quality of JPN and RUS defence against nage waza and katame waza did not differ significantly $(\mathrm{W}=143.0, \mathrm{p}=0.372$, $\mathrm{r}=-0.23$ [small effect] and $\mathrm{W}=128.0, \mathrm{p}=0.563, \mathrm{r}=-0.14$ [small effect]). Consequently, as in the case of Sa, there were significant differences in the So values between nage

Table 2. Efficiency of the throwing techniques ( $\mathrm{Sa}$ ) grouped by the Kodokan classification $[9,13]$

\begin{tabular}{lccccccccccc}
\hline & \multicolumn{2}{c}{ Te waza } & \multicolumn{2}{c}{ Koshi waza } & \multicolumn{2}{c}{ Ashi waza } & \multicolumn{2}{c}{ Ma sutemi $w$} & \multicolumn{2}{c}{ Yoko sutemi $w$} \\
\hline & JPN & RUS & JPN & RUS & JPN & RUS & JPN & RUS & JPN & RUS \\
\hline Mean & 1.3 & 0.74 & 0.23 & 0.60 & $\mathbf{3 . 7 2}$ & $\mathbf{1 . 6 4}$ & 0.21 & 0.13 & 0.13 & 0.44 \\
Median & 1.0 & 0 & 0 & 0 & $\mathbf{3 . 5 0}$ & $\mathbf{1 . 2 1}$ & 0 & 0 & 0 & 0 \\
SD & 1.42 & 1.06 & 0.51 & 1.07 & $\mathbf{2 . 1 3}$ & $\mathbf{1 . 5 7}$ & 0.37 & 0.50 & 0.49 & 0.79 \\
\hline
\end{tabular}

Bold type shows significant intergroup difference in ashi waza. Zero in the case of some of the techniques indicates that not all athletes used them.

Table 3. Efficiency of hold techniques ( $\mathrm{Sa}$ ) grouped by the Kodokan classification

\begin{tabular}{lcccccc}
\hline & \multicolumn{2}{c}{ Osaekomi waza } & \multicolumn{2}{c}{ Shime waza } & \multicolumn{2}{c}{ Kansetsu waza } \\
\hline Mean & JPN & RUS & JPN & RUS & JPN & RUS \\
Median & 1.03 & 0.505 & 0.0889 & 0.114 & 0.148 & 0.569 \\
SD & 0.714 & 0 & 0 & 0 & 0 & 0 \\
\hline
\end{tabular}

Zero in the case of some of the techniques indicates that not all athletes used them. 
Table 4. Quality of defence against nage waza and katame waza (So)

\begin{tabular}{lccc}
\hline Techniques & Mean & Median & SD \\
\hline Nage waza & & & \\
\hline JPN & 1.39 & 1.20 & 1.11 \\
RUS & 2.02 & 2.26 & 1.71 \\
\hline Katame waza & & & \\
\hline JPN & 0.05 & 0 & 0.18 \\
RUS & 0.13 & 0 & 0.36 \\
\hline
\end{tabular}

waza JPN and katame waza RUS $(\mathrm{r}=0.75)$ and between nage waza RUS and katame waza JPN.

Although the Kruskal-Wallis test showed significant differences in the efficiency in defence against throws $(\mathrm{H}=27.8$, $\mathrm{p}=0.008$, eta-squared $=0.11$ ), in the case of te waza alone there was a trend indicating worse defence in JPN than in RUS (W $=156.0 \mathrm{p}=0.091, \mathrm{r}=-0.38$ [large effect]. A negligible difference was found between JPN and RUS representatives for koshi waza $(\mathrm{W}=126.0, \mathrm{p}=0.777, \mathrm{r}=0.02)$, ashi waza $(\mathrm{W}=105.5, \mathrm{p}=0.557, \mathrm{r}=-0.04)$, ma sutemi waza $(\mathrm{W}=127.0, \mathrm{p}=0.617, \mathrm{r}=-0.13$ [small effect], and yoko sutemi waza $(\mathrm{W}=115.5, \mathrm{p}=0.829, \mathrm{r}=-0.06)($ Table 5$)$.

The Kruskal-Wallis test did not reveal significant differences when comparing individual subgroups of techniques used in defence against katame waza $(\mathrm{H}=3.002, \mathrm{p}=0.699$, eta-squared $=-0.07)$. Results for JPN and RUS representatives are shown in Table 6.

\section{The hierarchy of efficient techniques}

The hierarchy of techniques is presented in Tables 7 and 8. The most efficient technique for Japanese representatives during the World Championships in 2013, 2014 and 2015 was the foot throw uchimata, which is classified in the first place in all previously adopted criteria. For execution of this throw, athletes were most often awarded the ippon score (K1 indicator). It was also the most frequently assessed attack (K2 indicator), which resulted in the largest number of auxiliary points (K3 indicator). The next technique of Japanese athletes was the hand throw seoi nage, which took second place in all classification criteria. Out of 33 techniques efficiently used by Japanese representatives, the 10 most efficient are presented in Table 7.

The dominant technique for Russian athletes was the cross armlock ude hishigi juji gatame, taking first place in all the analysed criteria. The modified upper four-corner hold kuzure kami shiho gatame was inferior to the uchimata throw executed more frequently. Representatives of Russia efficiently used 30 techniques classified by Kodokan Judo, of which the best classified are shown in Table 8.

Table 5. Efficiency in defence (So) against attacks using nage waza throws

\begin{tabular}{lcccccccccc}
\hline & \multicolumn{2}{c}{ Te waza } & \multicolumn{2}{c}{ Koshi waza } & \multicolumn{2}{c}{ Ashi waza } & \multicolumn{2}{c}{ Ma sutemi $w$} & \multicolumn{2}{c}{ Yoko sutemi w } \\
\hline & JPN & RUS & JPN & RUS & JPN & RUS & JPN & RUS & JPN & RUS \\
\hline Mean & 0.14 & 0.58 & 0.25 & 0.23 & 0.83 & 0.93 & 0.03 & 0.09 & 0.14 & 0.18 \\
Median & 0 & 0 & 0 & 0 & 0.7 & 0 & 0 & 0 & 0 & 0 \\
SD & 0.31 & 0.78 & 0.52 & 0.37 & 0.96 & 1.80 & 0.13 & 0.30 & 0.26 & 0.40 \\
\hline
\end{tabular}

Zero in the case of some of the techniques indicates that not all athletes used them.

Table 6. Efficiency in defence (So) against attacks using katame waza holds

\begin{tabular}{lccc}
\hline Subgroup of techniques & Mean & Median & SD \\
\hline So Osaekomi JPN & 0.05 & 0 & 0.18 \\
So Osaekomi RUS & 0.08 & 0 & 0.29 \\
So Shime JPN & 0 & 0 & 0 \\
So Shime RUS & 0 & 0 & 0 \\
So Kansetsu JPN & 0 & 0 & 0 \\
So Kansetsu RUS & 0.06 & 0 & 0.23 \\
\hline
\end{tabular}

Zero in the case of some of the techniques indicates that not all athletes used them. 
Table 7. Dominant techniques among Japanese athletes during the World Championships in 2013, 2014 and 2015

\begin{tabular}{llccc}
\hline K rank & Name of the technique & K1 & K2 & K3 \\
\hline 1 & Uchimata (inner thigh reaping throw) & 1 & 1 & 1 \\
2 & Seoi nage (shoulder throw) & 2 & 2 & 2 \\
3 & Kuzure kesa gatame (modified scarf hold) & 2 & 4 & 4 \\
4 & Osoto otoshi (large outer drop) & 5 & 5 & 5 \\
5 & Osoto gari (large outer reap) & 4 & 6 & 6 \\
6 & Ouchi gari (large inner reap) & 12 & 3 & 3 \\
7 & Kouchi gari (small inner rep) & 8 & 6 & 7 \\
8 & Tate shiho gatame (straight four-corner hold) & 5 & 9 & 8 \\
8 & Juji gatame (cross armlock) & 5 & 9 & 8 \\
10 & Sumi otoshi (corner drop) & 12 & 8 & 10 \\
\hline
\end{tabular}

$\mathrm{K} 1$ - classification of the technique based on the number of ippon scores; K2 - classification of the technique based on the number of efficient attacks (assessed by judges' scores); K3 - classification of the technique based on auxiliary points scored for efficiently performed techniques (ippon - 10 points, waza ari -7 points, $y u k o-5$ points).

Table 8. Dominant techniques among Russian athletes during the World Championships in 2013, 2014 and 2015

\begin{tabular}{llccc}
\hline K rank & Name of the technique & K1 & K2 & K3 \\
\hline 1 & Ude hishigi juji gatame (cross armlock) & 1 & 1 & 1 \\
2 & Kuzure kami shiho gatame (modified upper four-corner hold) & 2 & 3 & 2 \\
3 & Uchimata (inner thigh reaping throw) & 3 & 2 & 3 \\
4 & Kouchi gari (small inner reap) & 3 & 3 & 4 \\
5 & Ouchi gari (large inner reap) & 3 & 3 & 5 \\
6 & Osoto gari (large outer reap) & 3 & 6 & 6 \\
6 & Seoi nage (shoulder throw) & 3 & 6 & 6 \\
8 & Kosoto gari (small outer reap) & 3 & 6 & 7 \\
9 & Kosoto gake (small outer hook) & 3 & 11 & 10 \\
9 & Deashi harai (forward foot sweep) & 3 & 11 & 10 \\
9 & Okuri eri jime (sliding collar strangle) & 3 & 11 & 10 \\
\hline
\end{tabular}

$\mathrm{K} 1$ - classification of the technique based on the number of ippon scores; K2 - classification of the technique based on the number of efficient attacks (assessed by judges' scores); K3 - classification of the technique based on auxiliary points scored for efficiently performed techniques (ippon - 10 points, waza ari -7 points, yuko -5 points).

\section{Discussion}

The main achievement of this study is that the values of indices of Japanese athletes' technical and tactical preparation, specifying the efficiency of both their attacks and their defence (the efficiency of their opponents' attacks), were higher than those of Russian representatives. Japanese athletes were characterised not only by greater efficiency of throwing techniques (nage waza) in comparison with Russian athletes, but also by their advantage in executing the techniques of holds (katame waza). In the group of throws from the "standing" position (tachi waza) Japanese athletes prevailed over Russian representatives in the efficiency of attack, while Russians held a slight edge over Japanese representatives in performing throws with tactical falls, the so-called "sacrifice throws" (sutemi wa$z a$ ). In addition, Japanese athletes demonstrated a greater efficiency in the use of foot techniques (ashi waza).

Representatives of Japan performed hand throws (te waza) and pinning (osaekomi waza) more efficiently than Russian athletes. Unlike Japanese representatives, 
Russians more efficiently used techniques less common in competitions from the group of locks (kansetsu waza), from the group of "sacrifice" throws with a fall to the side (yoko sutemi waza), and from the group of hip throws (koshi waza). In other groups of techniques, namely "sacrifice" throws with a fall on the back (ma sutemi waza, when the opponent leans forward), representatives of Japan were more efficient. In the group of chokes (shime waza), which were the least used, representatives of Russia demonstrated greater efficiency in attack.

Very similar characteristics of the training of athletes from both national teams were found in the analysis of the World Championship in 2010 and the Olympic Games in 2012 [3, 4]. This may suggest that competitors pursued different training programmes based on different technical groups.

The efficiency of defensive actions of Japanese and Russian athletes depended on the quality of tactical and technical actions executed by their opponents. It was found that Russian representatives' opponents could perform throws more efficiently than Japanese athletes' opponents. When applying holds, Japanese competitors were characterised by greater efficiency in defence, losing only a few points. When executing "standing" throws (tachi $w a z a$ ), also the opponents of Russian athletes were more efficient than opponents of the representatives of Japan. In the group of "sacrifice" throws (sutemi waza), Russian athletes' opponents attacked more efficiently. Opponents of both Russian and Japanese representatives used the technique from the group of foot throws (ashi waza) the most efficiently. Representatives of Japan showed greater efficiency in defence against hand throws (te waza) than Russian athletes. In other groups of techniques that were performed with less efficiency, representatives of Japan exhibited more efficiency in defence against hip throws (koshi waza) and "sacrifice" throws with a fall on the back (ma sutemi waza). Only in the group of "sacrifice" throws with a fall to the side (yoko sutemi waza) did representatives of Russia more efficiently hold off their opponents' attacks. In the group of holds (katame waza) JPN competitors exhibited high efficiency in defence, losing only a few points due to pinning (osaekomi waza). Representatives of Japan and Russia did not let their opponents efficiently implement chokes (shime waza).

Ranking of the techniques used by the compared teams significantly differed (Tables 7 and 8 ) and can attest to the different training systems that led to these teams' results in the Olympics in London and in Rio de Janeiro and in the world championships held between these Olympic Games (Table 9).

It follows from the previously conducted analysis and projections of a sports result that the profile of variability of achievements of the Japanese team, whose hallmark is a cyclical improvement in the result during the World Championships prior to the Olympic Games [27], should become a model of the judo training rhythm. The quality of the team's preparation could be increased using the value of individual indices assessing the conduct of a fight [4], after taking into account the contemporary training trends in different weight categories [29].

During the World Championships in 2013, 2014 and 2015, representatives of Japan were distinguished by a high index of the "efficiency in attack" with foot (ashi waza) and hand (te waza) throws, while in the group of holds (katame waza), they were by far the most efficient in using holds (osaekomi waza). The Japanese exhibited greater efficiency in attack and defence in throws from the group of tachi waza than Russian representatives. They had the biggest problems with opponents efficiently using throws from the "sacrifice" group (sutemi waza). Representatives of Japan manifested a similar profile of technical and tactical preparation during the World Championships prior to the Olympic Games in London, where their dominant techniques were also throws - uchimata and seoi nage [6]. High efficiency of foot throws (ashi waza) and the hand throw seoi nage repeatedly determined major successes of Japanese representatives during sports competitions, being a basis of their individual training tokui waza [5, 6, 23, 26, 30, 34]. The predominance of foot throws (ashi waza) also characterised Japanese athletes in

Table 9. Results obtained by the JPN and RUS teams in the Olympic Games and in the World Championships in the years 2012-2016 [33]

\begin{tabular}{|c|c|c|c|c|c|}
\hline $\begin{array}{l}\text { The number of medals } \\
\text { and scoring }\end{array}$ & $\begin{array}{c}\text { Olympic } \\
\text { Games (2012) }\end{array}$ & $\begin{array}{c}\text { World } \\
\text { Championships } \\
(2013)\end{array}$ & $\begin{array}{c}\text { World } \\
\text { Championships } \\
(2014)\end{array}$ & $\begin{array}{c}\text { World } \\
\text { Championships } \\
\text { (2015) }\end{array}$ & $\begin{array}{c}\text { Olympic } \\
\text { Games } \\
(2016)\end{array}$ \\
\hline $\mathrm{JPN}(\mathrm{G}, \mathrm{S}, \mathrm{B})$ & 4 & 4 & 4 & 7 & 7 \\
\hline$[\mathrm{pts}](4,2,1)$ & 6 & 13 & 11 & 18 & 14 \\
\hline $\operatorname{RUS}(\mathrm{G}, \mathrm{S}, \mathrm{B})$ & 5 & 2 & 7 & 2 & 2 \\
\hline [pts] $(4,2,1)$ & 15 & 2 & 9 & 4 & 8 \\
\hline
\end{tabular}

Medal achievements were recalculated into points $(\mathrm{G}=$ gold medal -4 points, $\mathrm{S}=$ silver medal -2 points, $\mathrm{B}=$ bronze medal -1 point $)$. 
the sports rivalry during the open championships of Japan [5]. In the World Championships in 2013, 2014 and 2015, representatives of Japan were distinguished by a high "efficiency in attack" index in foot (ashi waza) and hand (te waza) throws, while in the group holds (katame waza) by far they executed pinning (osaekomi waza) the best.

When representatives of Russia won three gold, one silver and one bronze medal in the men's judo tournament during the Olympic Games in London in 2012 it was a surprise to specialists. During this tournament there was a ban on holding the opponent's legs (in force since 2010), and such attacks constituted an important element in the technical-tactical preparation of Russian athletes $[7,8,15,16,24,31]$. The dominant techniques of Russian representatives during the analysed World Championships as well as during the previous Olympics (London 2012) were holds (katame waza), pinning (osaekomi waza) and armlocks (juji gatame) [7, 24]. In addition, values of efficient attacks (Sa) in other groups of techniques were similar during these events. Only the efficiency of attack of opponents of Russian representatives during the Olympic Games in London had significantly lower values. During the World Championships in 2013-2015, Russians' opponents obtained much higher values of the Sa index.

However, it should be remembered that a number of factors influence the efficiency of combat and the achieved sports results. These include morphological features, general preparation, psychomotor predispositions, as well as implementation of training programmes during which coaches and athletes make every effort to gain the optimum athletic form. The technical-tactical preparation of judo athletes is only one of the elements influencing the result of an efficient fight.

Such authors as Loison as well as Sacripanti and Pasculli present in their studies additional possibilities of analysing a judo fight that would allow other characteristics of judo athletes' technical-tactical preparation to be defined $[19,25]$. All this research enables improvement of the efficiency of preparing athletes for competition. Changes in the rules of judo sports fighting implemented since 2017 make it necessary to conduct further analyses of technicaltactical preparation of male and female athletes participating in competitive sports.

\section{Conclusions}

Summing up, during the analysed events, the representatives of Japan were characterized by greater efficiency of performing the techniques of throws and holds and by more efficient defensive actions than the representatives of Russia. Hence, it can be concluded that the results obtained by the two teams were associated not only with the efficiency of their defensive actions, but also with the type and efficiency of the techniques dominant in attack. The top two decisive techniques for Japanese representatives were throws (nage waza) - inner thigh reaping throw (uchimata) and shoulder throw (seoi nage) - while the top two techniques of Russian representatives were holds (katame waza): cross armlock (juji gatame) and modified upper four-corner hold (kuzure kami shiho gatame.

Conflict of interest: Authors state no conflict of interest.

\section{References}

1. Adam M (2013) Indices of technical-tactical preparation of judo athletes as a criterion for monitoring the level of training. Monografia habilitacyjna. Akademia Wychowania Fizycznego i Sportu w Gdańsku. [in Polish].

2. Adam M., M. Smaruj, R. Laskowski (2005) A graphic method of registering a judo fight. Sport Wyczynowy, 5/6: 33-43, [in Polish].

3. Adam M., M. Smaruj, R. Laskowski (2014) A technical and tactical profile of the double Olympic judo champion: a case study. Int. J. Sports Sci. Coach., 9(1): 123-138.

4. Adam M., M. Smaruj, R. Pujszo (2012) The individual profile of the technicatactical preparation of the World judo Champions in 2010-2011. Ido Movement for Culture. J. Martial Arts Anthrop., 12(2): 50-59.

5. Adam M., H. Tomita, M. Szymański, P. Klimowicz, S. Tyszkowski, B. Wolska (2015) Ways of performing judo throws, and their efficiency, assessed in the open weight category in All-Japan Judo Championships. Ido Movement for Culture. J. Martial Arts Anthrop., 15 (1): $39-45$.

6. Adam M., S. Tyszkowski, M. Smaruj (2011) The Contest Efficiency of the Men's National Judo Team of Japan and Character of Their Technical-Tactical Preparation during the World Judo Championships 2010. Balt. J. Health Phys. Act., 3 (1): 65-74.

7. Adam M., B. Wolska, P. Klimowicz, M. Smaruj (2013) Characteristics of technical-tactical preparation of Russian men's judo representation during the Olympic Games in London in 2012. Balt. J. Health Phys. Act., 5(4): 249-260.

8. Adams N., O.O. Yeoh (2011) Judo Evolution A guide to rule changes and Innovations. Ippon Books Ltd.

9. Anguera M.T., A. Blanco-Villasenor, J.I. Losada (2001) Observational Designs, Fundamental Key in the Process of Observational Methodology. Metodologia de las Ciencias del Comportamiento, (3): 135-160.

10. Anguera M.T., S. Izquierdo (2006) Methodological Approaches in Human Communication From Complexity of Situation to Data Analysis, [in:] Riva G, Anguera M.T, Mantovani F. Wiederhold H., (eds.), From 
Communication to Presence. Cognition, Emotions and Culture Towards the Ultimate Communicative Experience, IOS Press. Amsterdam., 203-222.

11. Cohen J. (1988) Statistical power analysis for the behavioural sciences. $2^{\text {nd }}$ ed. Hillsdale. NJ: Lawrence. Erlebum.

12. Cych P., W. Błach, Ł. Koleśnik, A. Levitskiy (2016) Error manifestations occur in junior and senior judo bouts full names. Journal of Combat Sports and Martial Arts, 1(7): 23-28.

13. Daigo T. (2005) Kodokan Judo Throwing Techniques. Kodansha International. Tokyo, New York, London.

14. Gutierrez-Santiago A., I. Prieto, O. Camerino T. Anguera (2011) The Temporal Structure of Judo Bouts in Visually Impaired Men and Women. J. Sports Sci., 29 (13): 1443-1451.

15. Hicks S., N. Soames (2001) 50 Great judo champions. Ippon Books.

16. Iatskevich A. (1999) Russian Judo. Ippon Books.

17. Kano J. (1994) Kodokan judo. Kodansha International. Tokyo, New York, London.

18. Kawamura T., T. Daigo (2000) Kodokan New JapaneseEnglish Dictionary of Judo. Kodokan.

19. Loison T. (2016) General analysis report - multi-factorial. http:/matchanalysis.fr/assets/files/a2ms.com.

20. Miarka B., D.H. Fukuda, F.B Del Vecchio, E. Francini (2016) Discriminant analysis of technical-tactical actions in high-level judo athletes. Int. J. Perform. Anal. Sport, 16: 30-39.

21. Miller G.A., N.A. Collins, M.J. Stewart, D.G. Callis (2015) Throwing technique and efficiency in the 2013 British Judo Championships. Int. J. Perform. Anal. Sport, 15: 53-68.

22. Nakamura M., Y. Takami, N. Masaki, K. Ito, N. Maekawa, M. Tamura (2014) Technical and tactical characteristic of Japanese high level women kendo players: comparative analysis. Archives of Budo, 10: 91-99.

23. Nakanishi H. (1992) Seoi-nage. Ippon Books.

24. Osipov A., M. Kudryavtsev, V. Struchkov, V. Kuzmin, A. Bliznevsky, I. Plotnikova (2016) Expert analysis of the competitive level of young Russian judo athletes who train for active attack fighting. J. Phys. Edu. Sport, 16(4): 1153-1158.

25. Sacripanti A., A. Pasculli (2009) Match analysis an undervalued coaching tool. Rotterdam. http:/arxiv.org/ abs/1004.1051v1.

26. Sato N. (1990) Ashiwaza. Ippon Books.

27. Sterkowicz S. (2000) A prognostic analysis of sports achievements of selected judo teams. Sport Wyczynowy, 11-12: 431-432, [in Polish]

28. Sterkowicz S., J.M. Garcia, F.S. Larma (2013) The importance of judo trainers' professional activities. Archives of Budo, 3(1): 57-61.

29. Sterkowicz-Przybycień K., B. Miarka, D.H. Fukuda (2017) Sex and weight category differences in timemotion analysis of elite judo athletes: Implications for assessment and training. J. Strength Cond. Res., 31(3): 817-825, DOI: 10.1519/JSC.0000000000001597.

30. Sugai H. (1991) Uchimata. Ippon Books.

31. Van de Walle R. (1993) Pick-Ups. Ippon Books.

32. Villasenor A.B., J.L. Losada Lopez, M.T. Anguera Arguilaga (2003) Data Analysis Techniques in Observational Designs Applied to the Environment-Behaviour Relation. Medio Ambiente y Comportamiento Humano, 4(2): 111-126.

33. www.ijf.org.

34. Yamashita Y. (1991) Osoto-gari. Ippon Books. Ltd. 55 Long Lane, London N3 2HY, England.

35. Yeoh O.O. (1993) Great judo championships of the world. Ippon Books Ltd. 55 Long Lane, London. N3 2HY, England.

\section{Received 12.12.2016 \\ Accepted 05.02.2018}

(c) University of Physical Education, Warsaw, Poland 